\title{
Trends in Incidence of Head and Neck Cancer in the Northern Territory, Australia, between 2007 and 2010
}

\author{
Rama Jayaraj ${ }^{1 *}$, Jagtar Singh ${ }^{1}$, Siddhartha Baxi ${ }^{3}$, Ramya Ramamoorthi ${ }^{1}$, \\ Mahiban Thomas ${ }^{2}$
}

\begin{abstract}
Incidence trends of head and neck cancer (HNC) have implications for screening strategies, disease management, guiding health policy making, and are needed to further oral cancer research. This paper aims to describe trends in age-adjusted HNC incidence rates focusing on changes across calendar period between 2007 and 2010 in Australian Northern Territory. Age-adjusted incidence rates of HNC were calculated for 20072010 using Northern Territory population based data assembled by Department of Health, Northern Territory Government of Australia. Changes in the HNC rate ratio (RR) and Estimated Annual Percentage Change (EAPC) between 2007-2008, 2008-2009 and 2009-2010 were calculated. A total of $171 \mathrm{HNC}$ patients were recorded by the Northern Territory Department of Health during the time period between 2007 and 2010, out of which, 135 were males (78.9\% of male HNC patients) and 36 were females (21.1\% of female HNC patients). In conclusion, HNC incidence rate has decreased in the Northern Territory Australian males but remains unchanged in Australian females. High incidences of HNC may be associated with the high smoking rate and high alcohol consumption in the Northern Territory. Continued monitoring of trends in HNC incidence rates is crucial to inform Northern Territory based cancer prevention strategies.
\end{abstract}

Keywords: Head and neck cancer - incidence - Northern Territory - tobacco smoking - alcohol - epidemiology

Asian Pac J Cancer Prev, 15 (18), 7753-7756

\section{Introduction}

Head and neck cancer (HNC) is the sixth most common cancer worldwide; annually 690,000 new cases were added; in the year 2012, global HNC rate was approximately $4.9 \%$ of total cancer incidence; In addition, approximately 375,000 people died from HNC in 2012, which was $4.6 \%$ of total cancer mortality (Braakhuis et al., 2014). The highest rate of age-adjusted incidence of HNC has reported in countries such as France, India, Brazil, and USA (Elango et al., 2006). In USA, approximately 36,500 new cases of HNC patients have diagnosed per year and 11,000 American deaths resulted from head and neck malignancies (Siegel et al., 2013). In Australia, HNC is the fifth most commonly occurring cancer and the incidence rate is about 12.3 per 100,000 inhabitants (Australian Institute of Health and Welfare, 2008). However, the high incidence rate of $\mathrm{HNC}$ has been previously reported in the Northern Territory (Cunningham et al., 2008).

The Northern Territory is situated in Central and Northern Central Australia and has a population of 233,300 that is spread over $1,350,000 \mathrm{~km}^{2}$. The Australian Aboriginal and Torres Strait Islander (Indigenous) constitute a substantial proportion of residents of the
Northern Territory (32\%) and are more likely to live in the remote communities. The Australian Indigenous cancer patients were diagnosed with very advanced stage of disease (HNC), and they have poorer survival rate than the non-Indigenous people (Cunningham et al., 2008). The early detection of disease and the appropriate therapy lead to successful treatment of HNC patients (Rezende et al., 2010).

Cancers of the head and neck comprise a heterogeneous grouping of tumours at several anatomical sites with different etiologic factors (Warnakulasuriya, 2009). A previous study reported that HNC is strongly associated with certain environmental and lifestyle risk factors including high tobacco smoking, high alcohol consumption and certain strains of Human Papilloma Virus (HPV) (Siegel et al., 2013). Tobacco smoking increases the risk of several cancers, including HNC (Basu et al., 2008). In Australia, approximately $70 \%$ of laryngeal cancers were attributed fully or partly due to smoking (Australian Institute of Health and Welfare, 2004). Excessive alcohol consumption can itself cause HNC or can act as a synergistic factor with tobacco smoking (Viswanathan and Wilson, 2004). In our previous reviews, we highlighted the major risk factors of $\mathrm{HNC}$ in

\footnotetext{
${ }^{124}$ School of Psychological and Clinical Sciences, Faculty of Engineering, Health, Science and the Environment, Charles Darwin University, ${ }^{2}$ Department of Head and Neck Surgery, Royal Darwin Hospital, Darwin, Northern Territory, ${ }^{3}$ Genesis Health Care Bunbury, Bunbury, Western Australia, Australia*For correspondence: Rama.Jayaraj@cdu.edu.au
} 
the Northern Territory (Singh et al., 2013; 2014).

In developing world, the incidence rate of HNC associated with tobacco and alcohol consumption has considerably decreased. However, HPV associated oro-oropharnygeal cancer has increased (Chaturvedi et al., 2009). Approximately 8,844 HPV associated oropharyngeal cancer patients were diagnosed between 1982 and 2005 in Australia (Hocking et al., 2011). This particular study demonstrated that the incidence of HPV associated HNC drastically increased per year for both males ( $1.42 \%$ annually) and females ( $1.04 \%$ annually) (Hocking et al., 2011). Intrapanya and colleagues reported that approximately 70 new cases of HNC were diagnosed between 2005 to 2009 at the Royal Darwin Hospital, Northern Territory, Australia (Intrapanya et al., 2012). However, the incidence rate of HNC has reported to be the same for Indigenous and non-Indigenous people, but mortality rate of $\mathrm{HNC}$ among Indigenous people was three times higher than non-Indigenous people (Moore et al., 2010). Indigenous people comprise only $2.4 \%$ of the Australian population, but high proportion of $(32 \%)$ of Indigenous people are residing in the Northern Territory (Pink, 2006). This particular segment of the Northern Territory accounted for over $47 \%$ of the HNC presenting to the Royal Darwin Hospital (Thomas et al., 2011). However, there were not enough studies conducted to demonstrate the trends in incidence rate of $\mathrm{HNC}$ in the Northern Territory population over the period of time. This paper aimed to update the changes of $\mathrm{HNC}$ incidence trends from 2007 to 2010 in the Northern Territory.

\section{Materials and Methods}

The HNC incidence data was collected from Department of Health, Northern Territory Government of Australia. HNC cases were classified as per the "International Classification Disease" (ICD) coding system of World Health Organisation (WHO) (Dobrossy, 2005). The term "International Classification Disease for Oncology" (ICD-O) often used at Royal Darwin Hospital at the time of diagnosis of sub-types of HNC. The ICD code for HNC (C01-C14 and C30-C32) is a term given to epithelial malignancies in the oral cavity (C01-C09), pharynx (C10-C14), nasal cavity (C30), para-nasal sinuses (C31) and larynx (C32). The Northern Territory Department of Health often uses the "International Agency for Research on Cancer" (IARC) and the "International Association of Cancer Registries" rules to code all cancer diagnoses (Fritz et al., 2000).

For this study, the measures including age-adjusted HNC incidence rates, over period of time, Rate Ratio (RR) and Estimated Annual Percentage Change (EAPC) were calculated. Incidence rates of HNC are generally expressed per 100,000 populations. We have collected the HNC incidence data for four years between 2007 and 2010 and estimated the age-adjusted rates for male and female population. The statistical results from these models include Estimated Annual Percentage Change (EAPC), 95\% confidence intervals (CIs) for the EAPCs, and any possible join points in year at which there was a change in the trends of HNC.

\section{Results}

Changes in HNC incidence rates from 2007 to 2010 varied substantially for male population of Northern Territory. The age-adjusted HNC incidence rates for Northern Territory were presented in (Table 1). The Northern Territory Department of Health recorded a total of $171 \mathrm{HNC}$ patients during the time period 2007 to 2010. Figure 1 showed the trends of new HNC patients between 2007 and 2010 in the Northern Territory.

According to the HNC data for 2007, the overall incidence rate of $\mathrm{HNC}$ in the Northern Territory population found to be 31.1 per 100, 000. This figure was far higher than the rest of Australian States and Territories. In year 2007, the incidence of HNC in males was observed about 52 per 100,000 with 42 new cases and HNC incidence rate in the females was 10.2 per 100,000 with 10 new cases. It was approximately 5 times higher in males than in females.

In 2008, the overall $\mathrm{HNC}$ incidence slightly decreased and estimated approximately 21.9 per 100,000 , whereas males reported approximately 30.5 per 100,000 with 33 new cases diagnosed and HNC incidence rate in females found to be 12.5 per 100,000 with 9 new cases. The HNC incidence were almost similar between 2009 and 2010 approximately 25 per 100,000 and 21 per 100,000 respectively. In 2009 , thirty three new male $\mathrm{HNC}$ patients were diagnosed and the rate of $\mathrm{HNC}$ incidence was 33.5 per 100,000. In females, nine new HNC cases were identified and incidence rate was 14.7 per 100,000 . The incidence rate of $\mathrm{HNC}$ in males was 31.8 per 100,000 with 36 new $\mathrm{HNC}$ cancer patients diagnosed and the rate

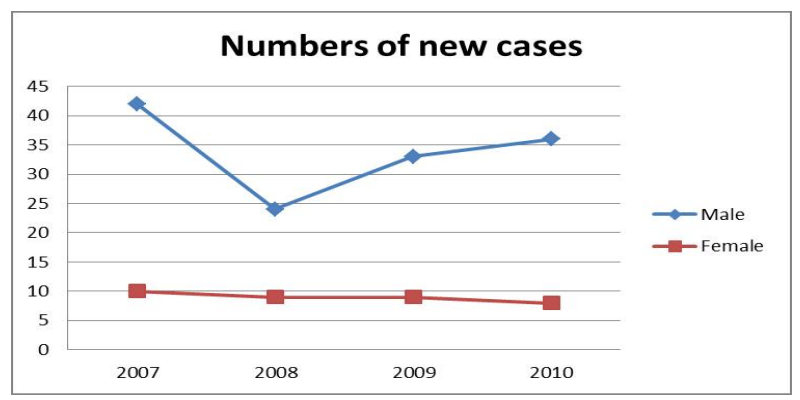

Figure 1. New Cases of HNC in The Northern Territory by Sex, 2007-2010

Table 1.Age-Adjusted Incidence Rates and Confidence Interval of HNC by Sex and Years

\begin{tabular}{lccr}
\hline $\begin{array}{l}\text { Year of } \\
\text { diagnosis }\end{array}$ & Sex & $\begin{array}{c}\text { Age-adjusted } \\
\text { incidence rate/ } \\
100,000\end{array}$ & $(95 \% \mathrm{CI})$ \\
\hline 2007 & Male & 52 & $(34.0-70.0)$ \\
& Female & 10.2 & $(3.8-16.6)$ \\
2008 & Total & 31.1 & $(17.5-35.5)$ \\
& Male & 30.5 & $(16.2-44.8)$ \\
& Female & 12.4 & $(3.4-21.4)$ \\
2009 & Total & 21.9 & $(13.4-30.3)$ \\
& Male & 33.5 & $(21.2-45.9)$ \\
2010 & Female & 14.7 & $(3.6-25.8)$ \\
& Total & 25 & $(16.5-33.5)$ \\
& Male & 31.8 & $(21.2-42.4)$ \\
& Female & 8.2 & $(12.4-14.0)$ \\
\hline
\end{tabular}

CI: 95\% Confidence intervals; Incidence rates per 100,000 age-adjusted 
Table 2. Rate Ratio and Estimated Annual Percentage Changes by Sex and Years

\begin{tabular}{ccccccc}
\hline \multicolumn{4}{c}{ Male } & \multicolumn{2}{c}{ Female } & \multicolumn{2}{c}{ Total } \\
& Rate Ratio & EAPC & Rate Ratio & EAPC & Rate Ratio & EAPC \\
\hline $2007-2008$ & 0.58 & -42.85 & 1.21 & 24 & 0.7 & -36.53 \\
$2008-2009$ & 1.09 & 37.5 & 1.18 & -27.41 & 1.14 & 27.27 \\
$2009-2010$ & 0.94 & 9.09 & 0.55 & -11.11 & 0.84 & 4.76 \\
\hline
\end{tabular}

Rate ratio comparing 2007-2008, 2008-2009 and 2009-2010; EAPC: estimated annual percentage changes

in the females was reported 8.2 per 100,000 with 8 new cases in 2010.

The RR and EAPC of HNC among males and females between 2007 and 2010 of the Northern Territory were presented in (Table 2). An increasing RRs was observed among females ( $R R=1.21$ and 1.18 respectively) than males (0.58 and 1.09 respectively) from 2007 to 2008 and from 2008 to 2009. However, Rate Ratio in males were noticed with higher $(R R=0.94)$ than the females $(\mathrm{RR}=0.55)$ from 2009 to 2010.

\section{Discussion}

Our analysis of Northern Territory HNC incidence rates showed heterogeneity in trends based on gender and consecutive years. The HNC incidence rate of 2007 in males was slightly higher than other three years. However, the HNC incidence was almost similar from 2008 to 2010. In females, the incidence of HNC was slightly higher in 2009 than other three years (2007, 2008 and 2010). Interestingly, the overall incidence of HNC rate has slightly decreased after 2007 in the Northern Territory, but higher rate compared to other Australian states and Territories. The incidence rate was approximately 32.78 per 100,000 among Australian Indigenous population (Thomas et al., 2011) due to the high consumption of tobacco and excessive usage of alcohol consumption in these communities (Jayaraj et al., 2012). The high incidence of HNC in the Northern Territory Indigenous people indicated an urgent need to improve early screening and diagnosis of the disease among this population.

Previous studies reported that tobacco use was considered as the strongest risk factor for $\mathrm{HNC}$, especially for oro - oropharyngeal cancers (Sturgis et al., 2004). The high alcohol consumption also act as major risk factor for HNC in many countries such as Hungary and other Central and Eastern European countries where alcohol consumption has increased or stabilised at high levels (Ezzati and Riboli, 2013). In 2007 and 2008, the Northern Territory had high prevalence of smoking (50\%) among Indigenous people and $28 \%$ among non-Indigenous population (Australian Bureau of Statistics, 2008). The tobacco smoking rate was measured as the highest in the Northern Territory compared to any States or Territories in Australia (Skov et al., 2010). In the Northern Territory, the per capita alcohol consumption of was approximately 15 litres per year of pure alcohol, which was considerably very high compared to the national level (Jayaraj et al., 2012).

Globally, the incidence rate of oropharyngeal cancers was $25 \%$ due HPV infection and $60 \%$ rate of HPV associated HNC was also noticed in few economically developed countries such as United States and France (Australian Government and Cancer Registry, 2006). Our previous research highlighted that the incidence and clinical outcomes of HPV associated oropharyngeal malignancies in the Northern Territory (Intrapanya et al., 2012). In this study, preliminary findings indicated that $45 \%$ (26 out of 57) of HNC patients tested were positive for p16 and $63 \%$ of those positive were Indigenous Australians (Intrapanya et al., 2012). Our data in this paper have shown that there was little improvement in the incidence of HNC among male population in the Northern Territory. However, there was not adequate improvement in the incidence rate of $\mathrm{HNC}$ among female population.

Before attempting to interpret the trends of $\mathrm{HNC}$, it was very important to evaluate the reliability of the incidence data. Our clinical research team has faced significant difficulties to in estimate HNC trends because there was not complete and reliable data available in the Department of Health, Northern Territory, particularly for Indigenous Australian population. In Indigenous Australians, the new cases of HNC in the Northern Territory might also be misjudged as some patients were living in very rural and remote communities. There would be high probability to underestimate the accurate number of non-Indigenous HNC patients, because some of HNC patients tend to travel interstate for treatment, particularly those with private health insurance. The above limitations representing that there was a big gap between cancer epidemiological research and the evaluation of Northern Territory HNC incidence rate; thus, further research in this context is warranted.

The high $\mathrm{HNC}$ incidence rate is a big concern in the Northern Territory. The high incidence of HNC might be associated with high prevalence of tobacco smoking and alcohol consumption in the Northern Territory. Although, there was moderate decrease of HNC incidence among the male patients after 2007 , female HNC patients were highlighted with no big changes in HNC incidence rates. Remarkably, the incidence of HNC in the Northern Territory is comparatively higher than other Australian States and Territories. There is a dearth of data pertaining to the incidence for HNC patients in the Northern Territory. Despite the foregoing limitations, we believe that our collected data could represent the most comprehensive summary of the current trends of HNC in the Northern Territory, Australia.

\section{Acknowledgements}

The research is supported by School of Psychological and Clinical Sciences, Charles Darwin University, Northern, Territory, Australia.

\section{References}

Australian Bureau of Statistics (2008). National Aboriginal and Torres Strait Islander Social Survey. Canberra: ABS cat.no.4714.0.

Australian Government and Cancer Registry (2006). Cancer in Australia 2006. Canberra: Australia Government Cancer Registry. 
Australian Institute of Health and Welfare (2004). Cancer in Australia 2001:Cancer series no. 28. Canberra: AIHW cat No. CAN 23.

Australian Institute of Health and Welfare (2008). Cancer in Australia: an overview 2008. Canberra: Australasian Association of Cancer Registries.

Basu R, Mandal S, Ghosh A, et al (2008). Role of tobacco in the development of head and neck squamous cell carcinoma in an eastern Indian population. Asian Pac J Cancer Prev, 9,381-6.

Braakhuis BJ, Leemans CR, Visser O (2014). Incidence and survival trends of head and neck squamous cell carcinoma in the Netherlands between 1989 and 2011. Oral Oncol, 50, 670-5.

Chaturvedi AK, Madeleine MM, Biggar RJ, et al (2009). Risk of human papillomavirus-associated cancers among persons with AIDS. J Natl Cancer Inst, 101, 1120-30.

Cunningham J, Rumbold AR, Zhang X, et al (2008). Incidence, aetiology, and outcomes of cancer in Indigenous peoples in Australia. Lancet Oncology, 9, 585-95.

Dobrossy L (2005). Epidemiology of head and neck cancer: magnitude of the problem. Cancer Met Rev, 24, 9-17.

Elango JK, Gangadharan P, Sumithra S, et al (2006). Trends of head and neck cancers in urban and rural India. Asian Pac $J$ Cancer Prev, 7, 108.

Ezzati M, Riboli E (2013). Behavioral and dietary risk factors for noncommunicable diseases. N Engl J Med, 369, 954-64.

Fritz A, Percy C, Jack A, et al (2000). International Classification of Diseases for Oncology. World Health Organisation: Geneva.

Hocking J, Stein A, Conway E, et al (2011). Head and neck cancer in Australia between 1982 and 2005 show increasing incidence of potentially HPV-associated oropharyngeal cancers. Br J Cancer, 104, 886-91.

Intrapanya M, Jayaraj R, Scott C, et al (2012). Human papilloma virus in oropharyngeal cancers (Re: ANZ J. Surg. 2011; 81: 581-3). ANZ J Surg, 82, 189-90.

Jayaraj R, Thomas M, Thomson V, et al (2012). High risk alcohol-related trauma among the Aboriginal and Torres Strait Islanders in the Northern Territory. Subst Abuse Treat Prev Policy, 7, 33.

Moore S, O'Rourke P, Mallitt K, et al (2010). Cancer incidence and mortality in Indigenous Australians in Queensland 19972006. Med J Aust, 193, 590-3.

Pink B (2006). Population distribution, Aboriginal and Torres Straits Islander Australian Bureau of statistics 47050.

Rezende TM, de Souza Freire M, Franco OL (2010). Head and neck cancer: proteomic advances and biomarker achievements. Cancer, 116, 4914-25.

Siegel R, Naishadham D, Jemal A (2013). Cancer statistics, 2013. CA Cancer J Clin, 63, 11-30.

Singh J, Jayaraj R, Baxi S, Ramamoorthi R, Thomas M (2013) Incidence and mortality from mucosal head and neck cancers amongst Australian states and territories: what it means for the northern territory. Asian Pac J Cancer Prev, 14, 5621-4..

Singh J, Ramamoorthi R, Baxi S, et al (2014). The risk factors of head and neck cancer and their general patterns in Australia: a descriptive review and update. J Environ Pathol Toxicol Oncol, 33, 45-57.

Skov SJ, Chikritzhs TN, Li SQ, et al (2010). How much is too much? Alcohol consumption and related harm in the Northern Territory. Med J Aust, 193, 269-72.

Sturgis E, Wei Q, Spitz M (2004). Descriptive epidemiology and risk factors for head and neck cancer. Semin Oncol, 31, 726-33.

Thomas M, Singh J, Scott C, et al (2011). Head and Neck Malignancy In The Northern Territory. Int J Head Neck
Surg, $4,2$.

Viswanathan H, Wilson JA (2004). Alcohol - the neglected risk factor in head and neck cancer. Clin Otolaryngol Allied Sci, 29, 295-300.

Warnakulasuriya S (2009). Global epidemiology of oral and oropharyngeal cancer. Oral Oncol, 45, 309-16. 